\title{
NOVEL IMPLEMENTATION OF TRANSVERSAL FILTERS IN MULTILAYERED MICROSTRIP TECHNOLOGY
}

\author{
D. C. Rebenaque, J. P. García, F. Q. Pereira, J.L.G. Tornero \\ and A. A. Melcon \\ Technical University of Cartagena \\ Department of Information Technologies and Communications \\ Campus Muralla del Mar, Ed Antigones, Pz. Hospital 1 \\ 30202 Cartagena, Murcia, SPAIN
}

\begin{abstract}
This paper presents novel and compact implementations of bandpass filters using transversal topologies. The structures presented implement different transfer functions of order three in multilayered microstrip technology. In order to allow for easy implementation of the required coupling, multilayered broadside configurations are proposed. We demonstrate that with the proposed configurations, both dualband and quasi-elliptic responses can be easily synthesized, by only changing the sign of certain entries in the coupling matrix. To easily adjust the sign of the coupling, novel resonators are proposed, including simple half-wavelength transmission line resonators, meander line resonators and shortcircuited resonators. Experimental validations for both prototypes are presented, demonstrating the validity and usefulness of the proposed configurations.
\end{abstract}

\section{INTRODUCTION.}

The deployment of new communication systems with increased bandwidth allocations requires the use of microwave filters with enhanced characteristics like multiband operation or sharp transition slopes. In particular, it must be taken into consideration for new applications the selectivity of the filter, and the ability to reject unwanted spurious signals that may interfere in the crowded electromagnetic spectrum [1].

Also, the increasing difficulties in the selection of communication channels, has fostered research in new multiband filters. Recent contributions of microwave filters have been focused on the synthesis and design of elliptic or pseudoelliptic filters for channel separation 
or multiband structures $[2,3,4]$. The insertion of transmission zeros at finite frequencies provides the necessary sharp cutoff slopes and reconfigurability responses, depending on the requirements of the application. In particular, synthesis techniques available for the design of filtering structures, implementing transmission zeros, could be based in a main structure with an in-line coupling topology, to which additional bypass or cross coupling between nonadjacent resonators are introduced $[5,6]$.

One important research line for filter design in the last years has been the development of transversal coupling topologies. This has been motivated by the availability of an exact synthesis technique of the generalized coupling matrix, after the work presented in [7]. In this topology, the input/output ports are coupled to all of the resonators of the filter, while all inter-resonator coupling values are zero. Thus, transmission zeros in the insertion loss response of the filter are produced due to the destructive interference of the signals that propagate along the different paths provided. One of the advantages of this topology is that it provides high flexibility to place a maximum number of transmission zeros in the insertion loss response of the filter. A wide class of frequency responses can be synthesized, ranging from multiband to quasi-elliptic responses. Normally, all these frequency responses can be easily implemented by adjusting the signs of certain elements of the coupling matrix.

On the contrary, a big disadvantage of the pure transversal topology, is that it is difficult to implement in practice many coupling paths from the input/output ports to all the resonators of the structure, still maintaining all other inter-resonator coupling values to zero. Resonators should be placed close to the ports to get the required coupling, whereas to preserve the transversal topology they should be isolated between them. Due to these difficulties, practical transversal implementations have been limited to low orders. For instance, a simple structure of order two can be found in [8]. Also, [9] shows an interesting example of a third order bandpass filter combining a microstrip dual-mode and a slot line resonators.

In this paper we propose alternative transversal microstrip filters of third order. As a difference with respect to the topology presented in [9] the resonators of the filter are implemented combining different types of printed lines, avoiding the use of slot resonators. To increase the number of coupling paths from the input/output ports to the resonators, a broadside coupling can be used in multilayer arrangements. To easily adjust the sign of the coupling, three different compact printed resonators are combined in the transversal structure, namely basic half wavelength printed line resonators, meander line 
resonators and central short-circuited stubs. We show that by combining appropriately all these types of resonators, several useful transfer functions can be effectively implemented. In this work we will show as an example the design of a multiband transversal filter and of a quasi-elliptic filter. Measured results of both prototypes are presented to show the validity of the new structures.

\section{NOVEL MICROSTRIP FILTERS}

The coupling and routing scheme of the third-order transversal filter, that will be designed in this paper, is shown in Fig. 1(b). The dark circles represent resonators, the source and the load are shown as empty circles, and the solid lines represent coupling between nodes. For comparison, in Fig. 1(a) we present a typical topology of a third order filter containing one trisection [10]. As it can be seen, in the transversal topology all resonators are directly coupled to the input/output ports, while all other inter-resonator coupling values are zero. The purpose of this section is to introduce new microstrip structures to implement this transversal coupling scheme.

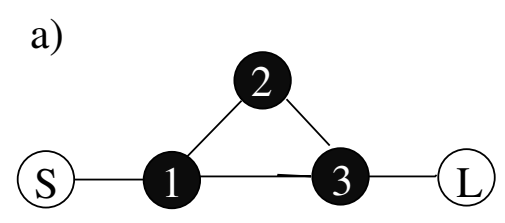

b)

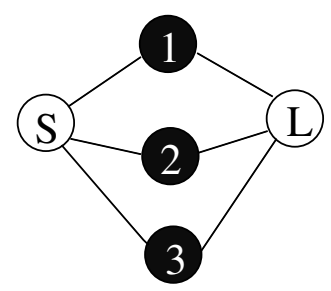

Figure 1. Coupling schemes: (a) Single trisection. (b) Transversal third order topology.

The first step in the design process of a filter is the calculation of the coupling matrix that synthesizes the desired frequency response. It should be noted that for the topologies presented in Fig. 1(b), a maximum of two transmission zeros at finite frequencies can be implemented. This can be inferred by the application of the minimum path rule [11]. For the pure transversal topology shown in Fig. 1(b), the coupling matrix can be easily calculated using the procedure presented in [7]. For multiband frequency responses, the procedure described in [12] can instead be used.

Once the coupling matrix has been computed, the next step is to synthesize the required coupling values with a suitable topology [13]. In the next subsections we present several examples using novel microstrip structures. These structures contain different kind of 
printed line resonators, and side-coupling [14] is combined with broadside coupling, when necessary, to provide for the required coupling paths. Two strategies are used to adjust the sign of the coupling values. The first is to use oversized resonators (meander line resonators) working at higher order resonances. The second is to use shortcircuited or open-ended terminations, as appropriate, to adjust the corresponding signs.

\subsection{Transversal filter with dual-band operation and two transmission zeros}

The first example corresponds to a pure transversal topology as shown in Fig.1(b), implementing a dual bandpass response. This topology can implement up to two transmission zeros at finite frequencies. By adjusting the sign of the coupling, different responses can be obtained, ranging from quasi-elliptic responses to dual-band responses. In the case of a dual band response, a transmission zero can be placed in-between the two passbands to increase rejection. The other transmission zero can be used to increase rejection at the other side of one of the passbands.

For the synthesis of the coupling matrix, the technique presented in [12], for dual-band transfer functions, has been used. The filter contains two bands, with a total bandwidth of $30 \mathrm{MHz}$ (lower passband) and $100 \mathrm{MHz}$ (upper passband), and return loss of $15 \mathrm{~dB}$. The coupling matrix obtained following the procedure described in [12] is $(1)$.

$$
M_{1}=\left(\begin{array}{rrrrr}
0 & -0.3498 & 0.4220 & -0.5675 & 0 \\
-0.3498 & 1.5961 & 0 & 0 & 0.3498 \\
0.4220 & 0 & -1.0990 & 0 & 0.4220 \\
-0.5675 & 0 & 0 & 0.0988 & 0.5675 \\
0 & 0.3498 & 0.4220 & 0.5675 & 0
\end{array}\right)
$$

For the practical implementation of this filter we select a microstrip structure with two dielectric substrates broadside coupled, as shown in Fig. 2. In the first substrate we print two resonators, together with the input/output lines. In the substrate on top we print the remaining resonator. In this way we can obtain a pure transversal topology of order three, with a very compact microstrip structure. We can observe in the $M_{1}$ coupling matrix (1), that the coupling of two resonators changes in sign from the input to the output port. This can be obtained by using simple $\lambda / 2$ printed line resonators. On the contrary, the sign of the coupling for the third resonator must be the same. To avoid the sign change, a printed line resonator can be 
Novel implementation of transversal filters in multilayered microstrip technology5

operated at the $\lambda$ resonance. This can be done easily by introducing a meander line resonator, as shown in Fig. 3.

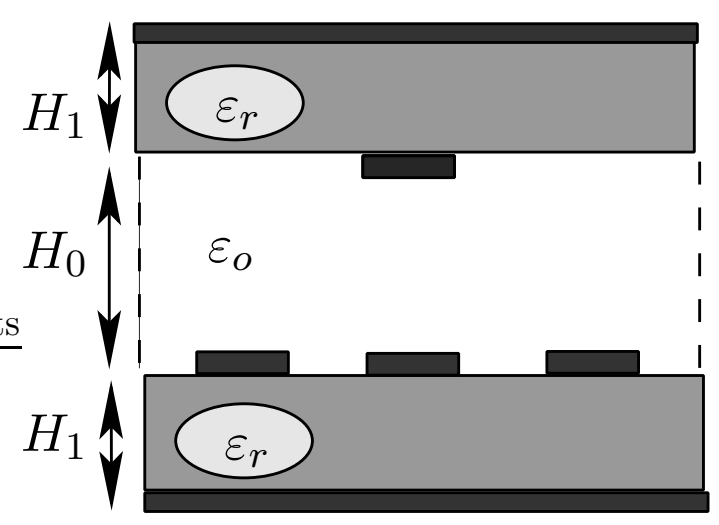

Figure 2. Lateral view of the proposed filter containing two broadside coupled dielectric substrates.

In Fig. 3 we show the basic topology used for the implementation of the filter in microstrip technology. The dotted resonator is broadside coupled to the input/output ports. To do this, the resonator is printed in the dielectric placed on top. The planar structure shown in Fig. 2 and Fig. 3 can be analyzed with the Momentum module of the commercial software Agilent-ADS. In Fig. 4 we present the results obtained from the analysis of the $M_{1}$ coupling matrix, together with the results obtained from the analysis of the multilayered structure, after the design process. We can observe very good agreement between the two responses. The dimensions obtained for this topology are shown in Table 1.

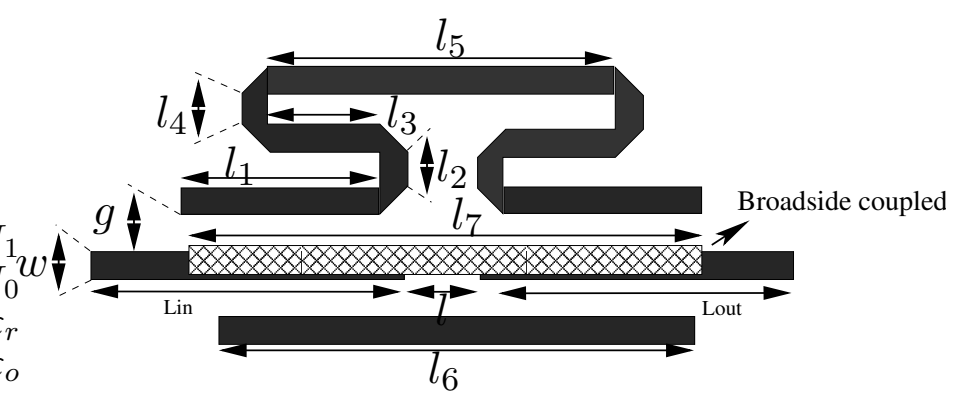

Figure 3. Layout of a third order transversal microstrip filter, implementing the coupling scheme of Fig. 1(b). 
6D. C. Rebenaque, J. P. García, F. Q. Pereira, J.L.G. Tornero and A. A. Melcon

Table 1. Dimensions of the dual-bandpass filter shown in Fig. 3.

\begin{tabular}{|c|c||c|c|}
\hline Dimension & Value (in mm.) & Dimension & Value (in mm.) \\
\hline \hline$w$ & 1.85 & $l_{1}$ & 20.8 \\
\hline$g$ & 0.3 & $l_{2}$ & 1.5 \\
\hline$H_{0}$ & 5.0 & $l_{3}$ & 13.2 \\
\hline$H_{1}$ & 1.27 & $l_{4}$ & 1.5 \\
\hline$\varepsilon_{r}$ & 6.15 & $l_{5}$ & 26.0 \\
\hline$L_{\text {in }}=L_{\text {out }}$ & 30.0 & $l_{6}$ & 51 \\
\hline$l$ & 5.0 & $l_{7}$ & 54 \\
\hline
\end{tabular}

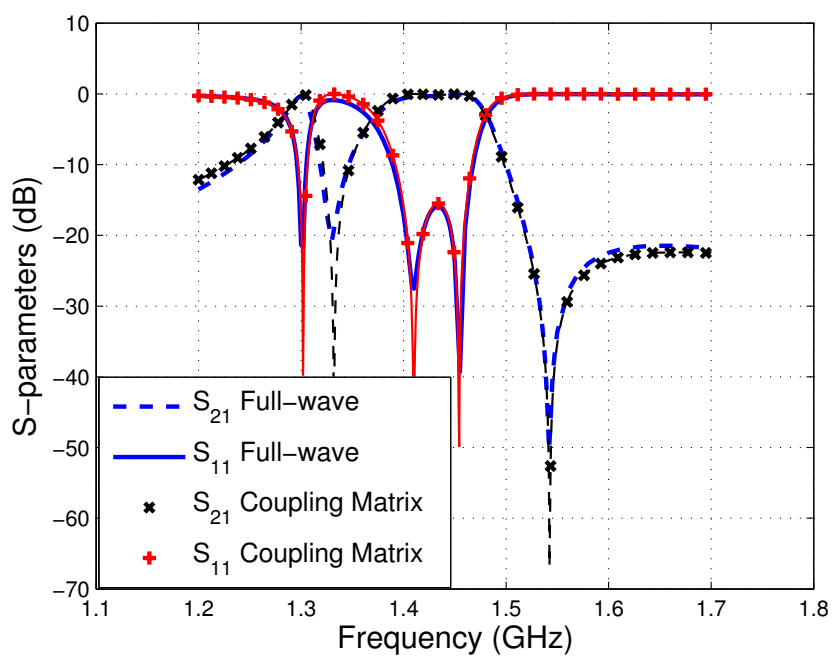

Figure 4. Fullwave simulations and coupling matrix response for the dual bandpass filter shown in Fig. 3.

\subsection{Transversal filter with pseudo-elliptic frequency response}

It is interesting to observe that the same configuration as introduced in the previous subsection can also be used to synthesize other types of frequency responses, for example, a single-band transfer function with quasi-elliptic response. The transmission zeros can be placed on both sides of the passband for maximum selectivity, by changing the sign of 
one coupling.

To illustrate the concept, we now design a single-bandpass filter with quasi-elliptic response and a bandwidth of $90 \mathrm{MHz}$. The two transmission zeros are located at $1340 \mathrm{MHz}$ and $1500 \mathrm{MHz}$, and the ripple level is $15 \mathrm{~dB}$. Since the topology corresponds to a transversal filter of order three, the synthesis procedure reported in [7] can be directly used to obtain the coupling matrix.

$$
M_{2}=\left(\begin{array}{rrrrr}
0 & 0.4236 & 0.4220 & -0.7128 & 0 \\
0.4236 & 1.190 & 0 & 0 & 0.4236 \\
0.4220 & 0 & -1.0990 & 0 & 0.4220 \\
-0.7128 & 0 & 0 & 0.0013 & 0.7128 \\
0 & 0.4236 & 0.4220 & 0.7128 & 0
\end{array}\right)
$$

We can see that the coupling matrix $M_{2}$ is very similar to the $M_{1}$ matrix previously computed. Only one of the resonators changes the sign of the coupling to the input port (compare second column of matrices $M_{2}$ and $M_{1}$ ). Also, the third resonator has to be adjusted (in coupling and length) to recover the desired response (see fourth column of the matrix).

The additional positive sign coupling can be implemented by changing the $\lambda / 2$ resonator in the upper substrate. However, it is not a convenient way to use a meander type resonator of length $\lambda$. This is because this resonator is located on the upper substrate, and besides the desired coupling between the terminal ports, it will easily introduce additional undesired cross-coupling to the other resonators of the structure. Thus, it is proposed an alternative configuration, based on maintaining the $\lambda / 2$ resonator, but placing a short-circuit in the central point of this line [15]. This short-circuit will force an even symmetry of the electric field in the resonator, so the sign will not change anymore from input to the output port.

In this way we can easily implement the same sign for the coupling elements $M_{S 1}=M_{L 1}=0.4236$. In Fig. 5 it is shown the new layout for the proposed microstrip configuration. Again, the dotted resonator is printed on the layer on top. The only modification of this structure with respect to the layout presented in Fig. 3, is the via-hole introduced at the central point of this resonator, used to implement the required short-circuit. Also, the other $\lambda / 2$ resonator has been adjusted by slightly shortening its length. It is important to point out that the meander resonator has not been changed, as indicated by the third column of matrix $M_{2}$, which is identical to the matrix $M_{1}$. The final dimensions for this filter are given in Table 2.

In Fig. 6 we present the results obtained from the analysis of the $M_{2}$ coupling matrix (2), together with full wave simulations of the 


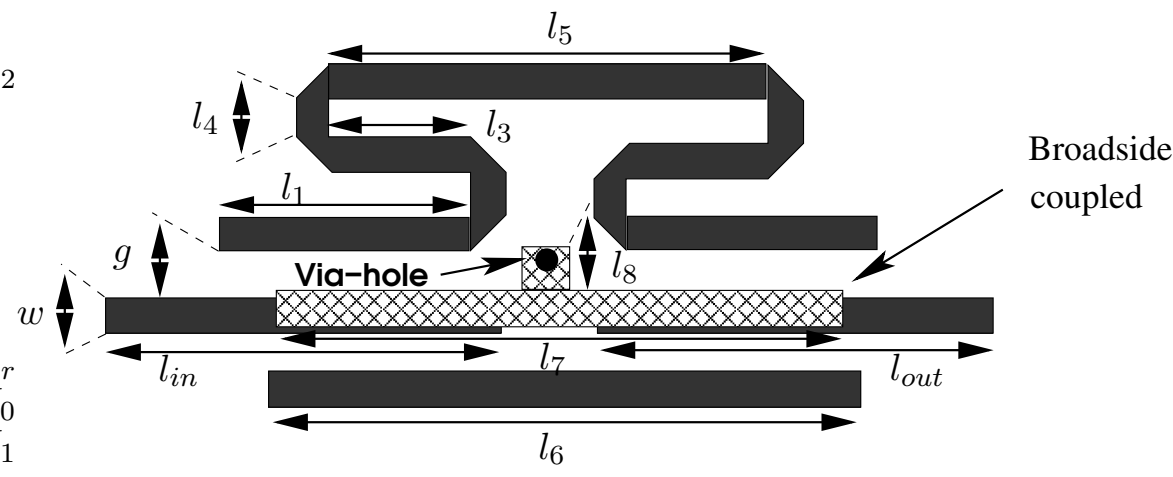

Figure 5. Layout of the third order transversal filter with quasielliptic response.

Table 2. Dimensions of the quasi-elliptic filter shown in Fig. 5.

\begin{tabular}{|c|c||c|c|}
\hline Dimension & Value (in mm.) & Dimension & Value (in mm.) \\
\hline \hline$w$ & 1.85 & $l_{2}$ & 1.5 \\
\hline$g$ & 0.3 & $l_{3}$ & 13.2 \\
\hline$H_{0}$ & 2.0 & $l_{4}$ & 1.5 \\
\hline$\varepsilon_{r}$ & 6.15 & $l_{5}$ & 26.0 \\
\hline$L_{\text {in }}=L_{\text {out }}$ & 30.0 & $l_{6}$ & 49.6 \\
\hline$l$ & 5.0 & $l_{7}$ & 45.4 \\
\hline$l_{1}$ & 20.8 & $l_{8}$ & 1.5 \\
\hline
\end{tabular}

structure shown in Fig. 5. We can observe good agreement between both responses. Only at the beginning of the passband, the full-wave simulation shows a slight deviation. It is believed that this is due to a spurious radiation of the via-hole at these frequencies.

\section{EXPERIMENTAL IMPLEMENTATION}

For a practical validation of the filter designs presented in this paper, both prototypes have been manufactured and tested in microstrip technology. The substrate selected for manufacturing is an $R T-$ Duroid/6006, with a relative permittivity $\epsilon_{r}=6.15$, and thickness $H_{1}=1.27 \mathrm{~mm}$. The circuit has been manufactured with an LPKF Protomat $60 \mathrm{C}$ drilling machine, and measurements are taken with an 


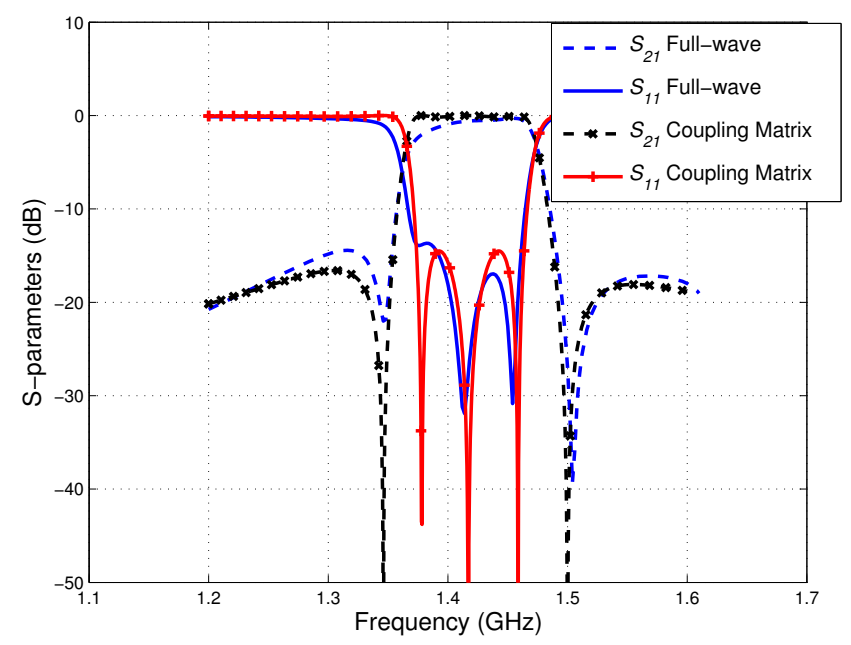

Figure 6. Fullwave simulations and coupling matrix response for the quasi-elliptic filter shown in Fig. 5.

HP-8714-ES vector network analyzer.

First, we will report the results obtained for the dual-bandpass filter. The basic layout of the fabricated filter is shown in Fig. 3 . In Fig. 7 we present the results obtained for the filter shown in Fig. 3, when losses are included in the simulations. Both, dielectric losses $(\tan \delta=0.0019)$ and losses in the printed metalizations $(\sigma=$ $\left.4 \cdot 10^{7} \Omega^{-1} / \mathrm{m}\right)$, are considered. Measured results are also included in Fig. 7 for comparison. We can observe good agreement between measured and predicted responses, for both passbands of the filter. The first passband shows an insertion loss of $2.7 \mathrm{~dB}$ and a return loss of $10 \mathrm{~dB}$, while the upper passband has a maximum insertion loss of $3 \mathrm{~dB}$ and return loss of $12 \mathrm{~dB}$.

The second filter showing quasi-elliptic response has also been fabricated and tested. Now, the layout of this filter is shown in Fig. 5. The results show a minimum insertion losses of $1.4 \mathrm{~dB}$ (simulated) and $1.05 \mathrm{~dB}$ (measured). The minimum return losses are $11.79 \mathrm{~dB}$ (simulated) and $9.11 \mathrm{~dB}$ (measured), and the measured bandwith is $100 \mathrm{MHz}$. The main differences are a small frequency shift of 20 $\mathrm{MHz}$, and the returns losses at the beginning of the passband. This discrepancy might be due to fabrication tolerances and to the spurious radiation of the via hole.

The manufactured prototypes are shown in Fig. 9 compared with 
10D. C. Rebenaque, J. P. García, F. Q. Pereira, J.L.G. Tornero and A. A. Melcon

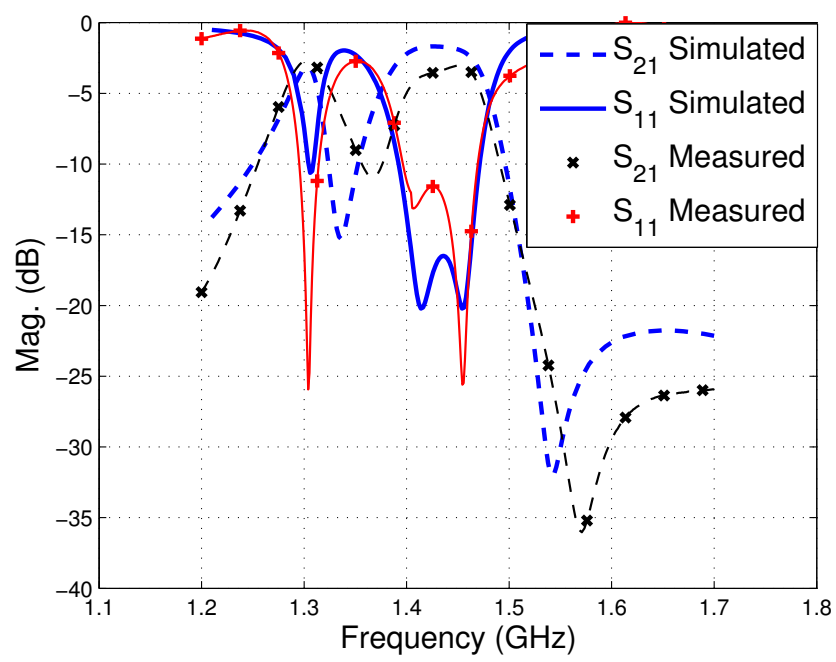

Figure 7. Comparison between simulated results and measured results for the filter shown in Fig. 3.

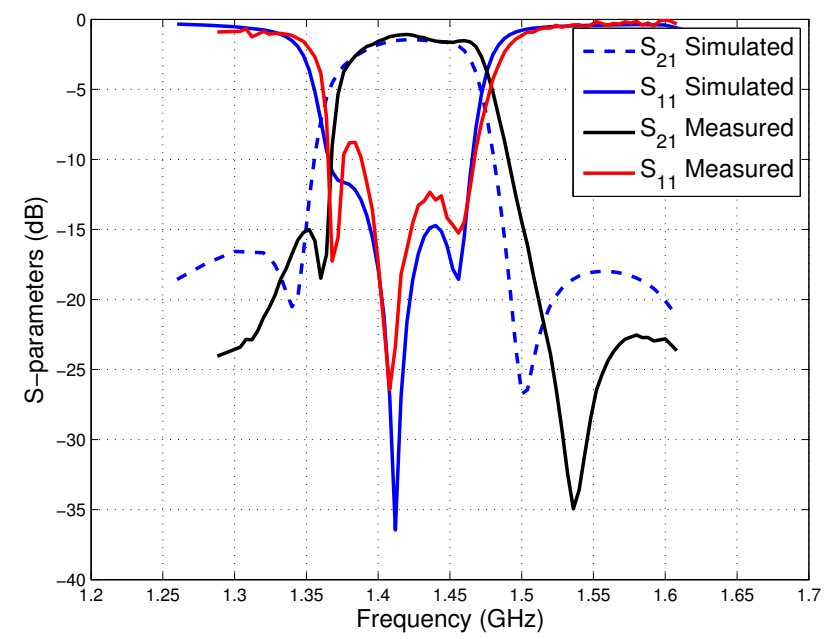

Figure 8. Comparison between simulated results and measured results for the filter shown in Fig. 5.

an Euro coin. The basic structure consists of two different dielectric 
substrates separated by a layer of air, providing the required broadside coupling to the input/output ports. The substrates are properly aligned to allow for a precise adjustment of this broadside coupling ( $H_{0}$ separation). The lower dielectric substrate of the filter contains the input/output ports and two of the resonators (one of type half wavelength and one of type meander line), and it can be seen on the left side of the photo. The meander line resonator implements the third column of the coupling matrices $\left(M_{1}\right)$ and $\left(M_{2}\right)$, while the half wavelength resonator implements the fourth column. Near the four corners of each substrate, holes are anticipated to allow for plastic screws, which are used for the correct alignment of both layers. Together with the plastic screws, nuts are also used to fix the correct separation of both substrates, required to implement the right values of the broadside coupling $\left(H_{0}\right)$. On the right side of the photo we show the upper dielectric substrates, containing the third resonator. This substrate (bottom) contains the basic printed line resonator, also of type half wavelength, needed to implement the second column of the coupling matrix $\left(M_{1}\right)$. For the second prototype, a via-hole is implemented at the center of this resonator as also shown in the photo (top), needed to implement the second column of the matrix $\left(M_{2}\right)$.

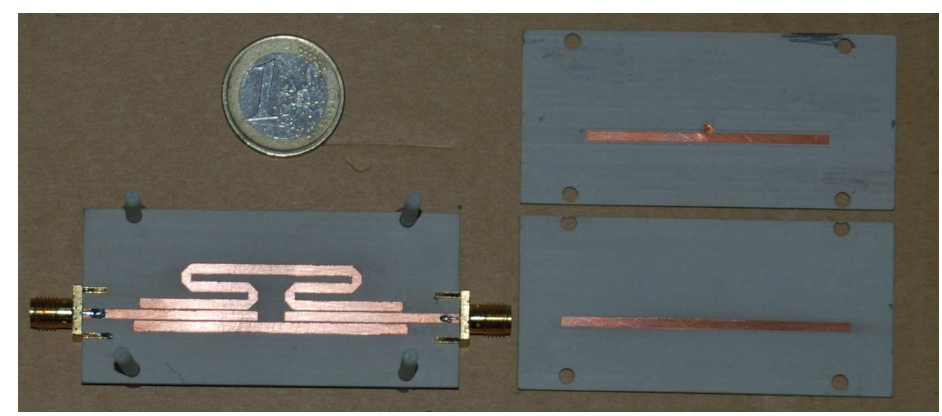

Figure 9. Photo of the manufactured prototypes.

\section{CONCLUSIONS}

Two third-order novel microstrip bandpass filters have been designed. The coupling schemes chosen correspond to transversal filters of order three, exhibiting two transmission zeros, and have been implemented in microstrip technology. It is shown that several frequency responses can be implemented, such as dual-band or quasi-elliptic. Several strategies to adjust the sign of the coupling values have been proposed. This includes the use of printed resonators of different lengths, and the 
introduction of short-circuits when appropriate. Measured results are presented for two prototypes. The obtained measurements show good agreement with respect to theoretical predictions, demonstrating the validity of the proposed structures.

\section{ACKNOWLEDGMENT}

The authors thank Ministerio de Educacion y Ciencia of Spain, which has supported this work with the Project TEC2007-67630-C03/TCM.

\section{REFERENCES}

1. Levy, R., Snyder, V. and Mattaei, G. "Design of microwave filters," IEEE Transactions on Microwave Theory and Techniques, Vol. 50, 783-793, March 2002.

2. Macchiarella, G. and Tamiazzo, S.. "Design techniques for dualbandpass filters," IEEE Transactions on Microwave Theory and Techniques, Vol. 53, no 11, 3265-3271, November 2005.

3. Dai, X. W., Liang, C. H., Wu, B. and Fan, J. W., "Novel dual-band bandpass filter design using microstrip open-loop resonators," Journal of Electromagnetic Waves and Applications, Vol. 22, 216$225,2008$.

4. Wu, G. L., Mu, W., Dai, X., W. and Jiao, Y. C. "Design of novel dual-band bandpass filter with microstrip meander-loop resonator and CSRR DGS," Progress in Electromagnetics Research, Vol. 78, 17-24, 2008.

5. Kurzok, R. M. "General four-resonator filters at microwave frequenciesfilters," IEEE Transactions on Microwave Theory and Techniques, Vol. 14, no 7, 295-296, July 1966.

6. Fan, J. W., Liang, C. H. and Dai, X. W. "Design of cross-coupled dual-band filter with equal length split-ring resonators," Progress in Electromagnetics Research, Vol. 75, 285-293, 2007.

7. Cameron, R., J. "Advanced coupling matrix synthesis techniques for microwave filters," IEEE Transactions on Microwave Theory and Techniques, Vol. 51, no 1, 1-10, January 2003.

8. Rebenaque, D., C., Pereira, F. D., Garcia, J., P., Melcon, A., A. and Guglielmi, M. "Two compact configurations for implementing transmission zeros in microstrip filters," IEEE Microwave Wireless and Components Letters, Vol. 14, no 10, 475-477, October 2004.

9. Zhou, M., Tang, Z., and Xiao, F. "Compact transversal bandpass filter incorporating microstrip dual-mode open-loop resonator and 
slot line resonator with source-load coupling," Microwave and Optical Technology Letters, Vol. 51, no 12, 2927-2929, December 2009.

10. Garay, J., T., M. "Synthesis of physically asymmetrical ntrisection filters with transmission zeros at n-different real frequencies," Electronics Letters, Vol. 35, 226-227, February 1999.

11. Amari, S. and Bornemann, J. "Maximum number of finite transmission zeros of coupled resonator filters with source/loadmultiresonator coupling and a given topology," Proceeding of AsiaPacific Microwave Conference, 1175-1177, Sydney, Australia, 2000.

12. Mokhtaari, K., R., M., Bornemann, J. and Amari, S. "Couplingmatrix design of dual and triple passband filters," IEEE Transactions on Microwave Theory and Techniques, Vol. 54, no 11, 3940-3946, November 2006.

13. Swanson, D., and Macchiarella, G. "Microwave filter design by syntesis and optimization," Microwave Magazine, 67-69, April 2007.

14. Prabhu, J. W., Liang, C. H. and Dai, X. W. "Microstrip bandpass filter at S band using capacitive coupled resonator," Progress in Electromagnetics Research, Vol. 76, 223-228, 2007.

15. Razalli, M. S., Ismail, A., Mahdi, M. A. and Hamidon, M. N. "Novel compact microstrip ultra-wideband filter utilizing shortcircuited stubs with less vias," Progress in Electromagnetics Research, Vol. 88, 91-104, 2008. 